\title{
FILTERS FOR FAST 2D AND 3D PSEUDO-INVERSION OF THE RESISTIVITY PROFILES
}

\author{
Pietro Cosentino, Dario Luzio and Raffaele Martorana \\ Istituto di Geofisica Mineraria, University of Palermo \\ Via M. Stabile 110, 90139 Palermo (ITALY)
}

\begin{abstract}
Summary
The aim of the methodological approach here presented is to construct fast pseudosections starting from resistivity data: the pseudo-sections should match as closely as possible the geometry of the investigated structures.

In principle the procedure consists in arranging the experimental resistivity data in a sets of convolutions using 2D and 3D filters which can be easily calculated depending on the geometry of both the used electrode array and the range of the approximated investigation depths. The various filter coefficients can be also suitably corrected to produce different effects on the resulting pseudo-sections.

This method is discussed in some critical choice and some applications on synthetic data calculated on some simple models are presented. Even if the methodology is not a real inversion but a fast arrangement of the experimental data, it can be used as a useful tool in the interpretation, at least as a first step before an iterative inversion.
\end{abstract}

Key words: pseudosection, resistivity profile, IP profile, pseudo-depth section, pseudoinversion, electric tomography

\section{Introduction}

The concept of construct a resistivity or IP pseudosection was first introduced by Hallof (1957) for dipole-dipole array. The first proposal was to plot all the apparent resistivity (or IP) data collected along the profile at the point of intersection of two $90^{\circ}$ degree lines from the two dipole centers just below the center of the dipole-dipole array. Afterwards this concept has been extensively applied and it is in practice used by geophysicists till today over the world. This is because the construction of the pseudosection is very easy and fast to carry out, while the inversion of the resistivity and IP data is a very complex problem from the computing point of view, also for simple $2 \mathrm{D}$ models. Furthermore any inversion, due to its non linearity characteristics, demands a starting model, so that a fast pseudo-section construction can be a very useful tool also as a preliminary step to choose the starting model for the inversion.

Nevertheless this kind of pseudo-section technique neither gives an idea about the target depth nor means that the measured signal is coming from that depth (Apparao, 1991). Therefore many efforts have been made to improve the technique of construction of the pseudo-section and/or the way to extract information from it (Fraser, 1981, to recognize the main lateral discontinuities), especially the most requested one, i.e. the real depth of the detected anomalies. Edwars (1977) and Apparao (1991) produced interesting approach to change the scale of the pseudo-depths in the construction of the classic pseudo-sections with the aim of improving the recognition of the real pseudo-depths of the detected anomalies.

Afterwards Cosentino et al. (1995) and Moller et al. (1995) have presented two slightly different approaches of the 2D fast construction of the pseudo-sections which can be 
considered a significant innovation of the $2 \mathrm{D}$ interpretation.

In fact the former one is in practice based on a convolution of the experimental data:

$$
\rho(\mathrm{x}, \mathrm{y}, \mathrm{z})=\int g\left(\mathrm{x}-\tau_{1}, \mathrm{y}-\tau_{2}, \mathrm{z}\right) \rho_{\mathrm{a}}\left(\tau_{1}, \tau_{2}\right) \mathrm{d} \tau_{1} \mathrm{~d} \tau_{2},
$$

with $\mathrm{y}=0$ and $\tau_{2}=0$, which can be easily extended to 3D models by adding experimental data involving measurements with $y \neq 0$. The second one (Moller et al., 1995) present a fast deconvolution of the experimental data using the 2D Frechet kernel for the homogeneous halfspace.

\section{Tomographic pseudo-sections}

The $g\left(x-\tau_{1}, y-\tau_{2}, z\right)$ filter function of (1) has been calculated on the basis of an approximated influence factor of each pseudo-section pixel of the subsoil - centered on $(\mathrm{x}, \mathrm{y}, \mathrm{z})$ - on a resistivity measure carried out with a four electrode linear array, which has been calculated as:

$$
\begin{aligned}
I(x, y, z) & =\left[\left(x-x_{C 1}\right)\left(x-x_{P 1}\right)+y^{2}+z^{2}\right]\left\{\left[\left(x-x_{C 1}\right)^{2}+y^{2}+z^{2}\right]\left[\left(x-x_{P 1}\right)^{2}+y^{2}+z^{2}\right]\right\}^{-3 / 2}+ \\
& -\left[\left(x-x_{C 2}\right)\left(x-x_{P 1}\right)+y^{2}+z^{2}\right]\left\{\left[\left(x-x_{C 2}\right)^{2}+y^{2}+z^{2}\right]\left[\left(x-x_{P 1}\right)^{2}+y^{2}+z^{2}\right]\right\}^{-3 / 2}+ \\
& -\left[\left(x-x_{C 1}\right)\left(x-x_{P 2}\right)+y^{2}+z^{2}\right]\left\{\left[\left(x-x_{C 1}\right)^{2}+y^{2}+z^{2}\right]\left[\left(x-x_{P 2}\right)^{2}+y^{2}+z^{2}\right]\right\}^{-3 / 2}+ \\
& +\left[\left(x-x_{C 2}\right)\left(x-x_{P 2}\right)+y^{2}+z^{2}\right]\left\{\left[\left(x-x_{C 2}\right)^{2}+y^{2}+z^{2}\right]\left[\left(x-x_{P 2}\right)^{2}+y^{2}+z^{2}\right]\right\}^{-3 / 2},
\end{aligned}
$$

where $\left(\mathrm{x}_{\mathrm{C} 1}, 0,0\right)$ and $\left(\mathrm{x}_{\mathrm{C} 2}, 0,0\right)$ are the coordinates of the current electrodes and $\left(\mathrm{x}_{\mathrm{P} 1}, 0,0\right)$ and $\left(\mathrm{x}_{\mathrm{P} 2}, 0,0\right)$ those of the potential electrodes (formulas derived after Roy and Apparao, 1971).

For three of the main configurations used for the construction of the pseudo-sections (namely the linear symmetrical array of the $\alpha, \beta$ and $\gamma$ tri-potential measurements - see Habberjam, 1979) the influence coefficients have been calculated in a sufficiently large part of the overlying halfspace; some examples are presented in figures 1,2 and 3 using contour lines on a section passing trough the electrode line.

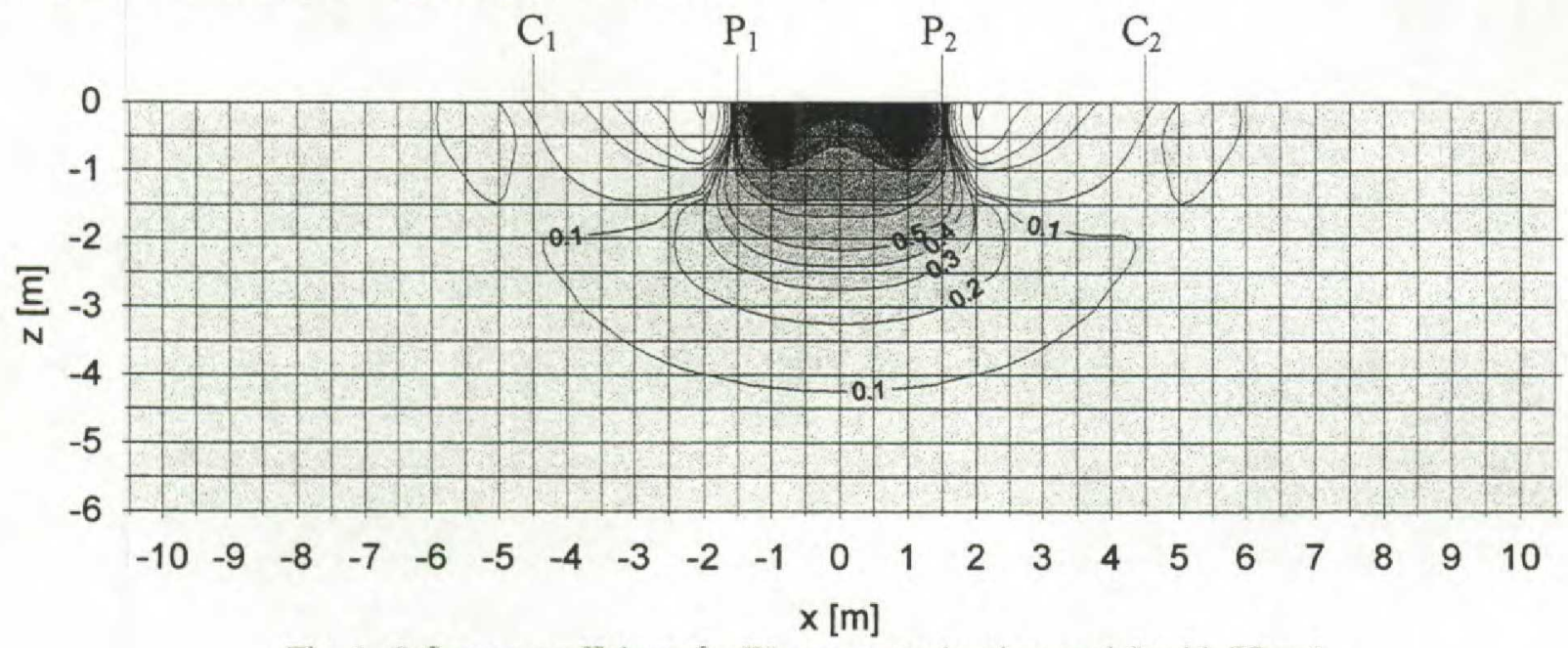

Fig. 1 - Influence coefficients for Wenner array ( $\alpha$ tri-potential) with $P L=6$.

It can be noticed that the influence coefficients for an apparent resistivity measurement are positive in a large part of the influence volume and are negative in some points of the subsoil, mostly located very close to some electrodes. If the coefficient is positive this means that an increment of resistivity in the pixel will produce an increment of the measured apparent resistivity: the negative coefficients will work in opposite way. 


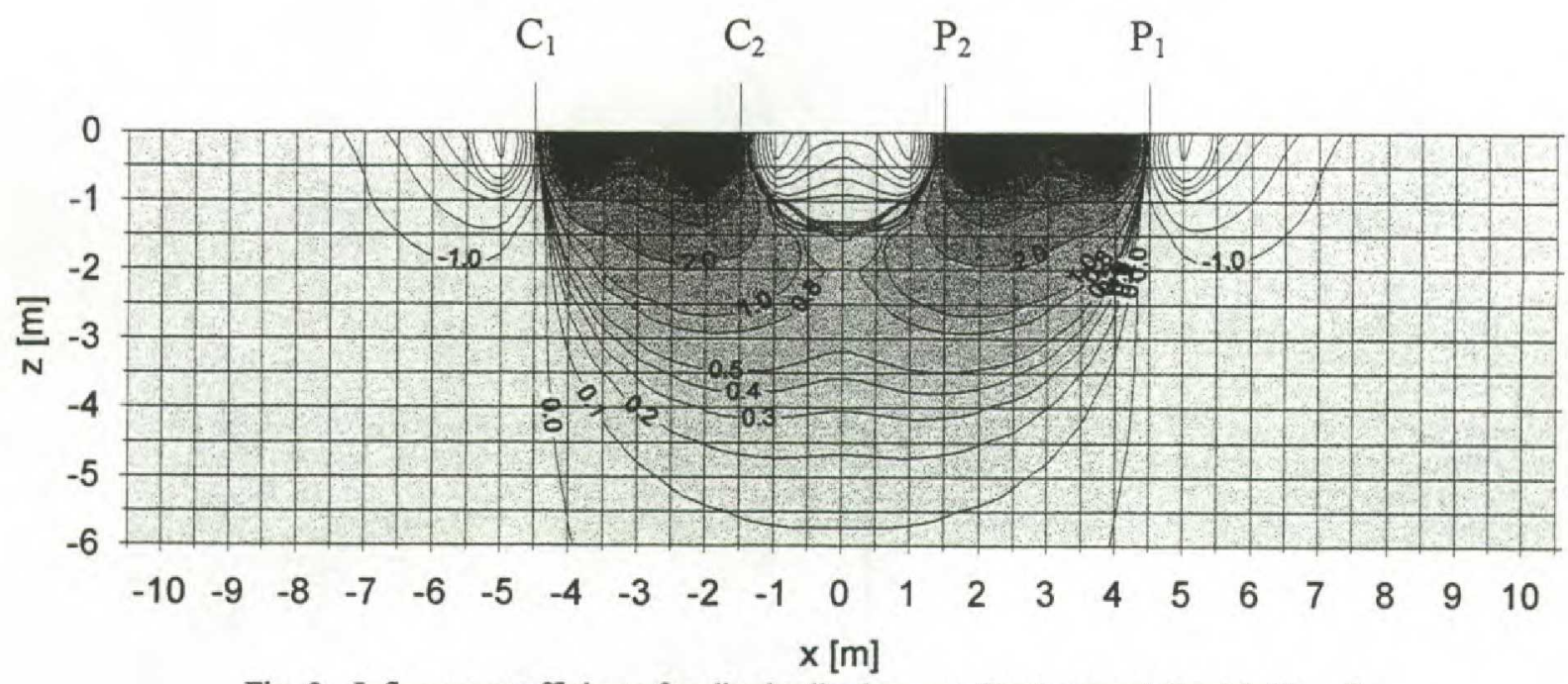

Fig. 2 - Influence coefficients for dipole-dipole array ( $\beta$ tri-potential) with $P L=6$.

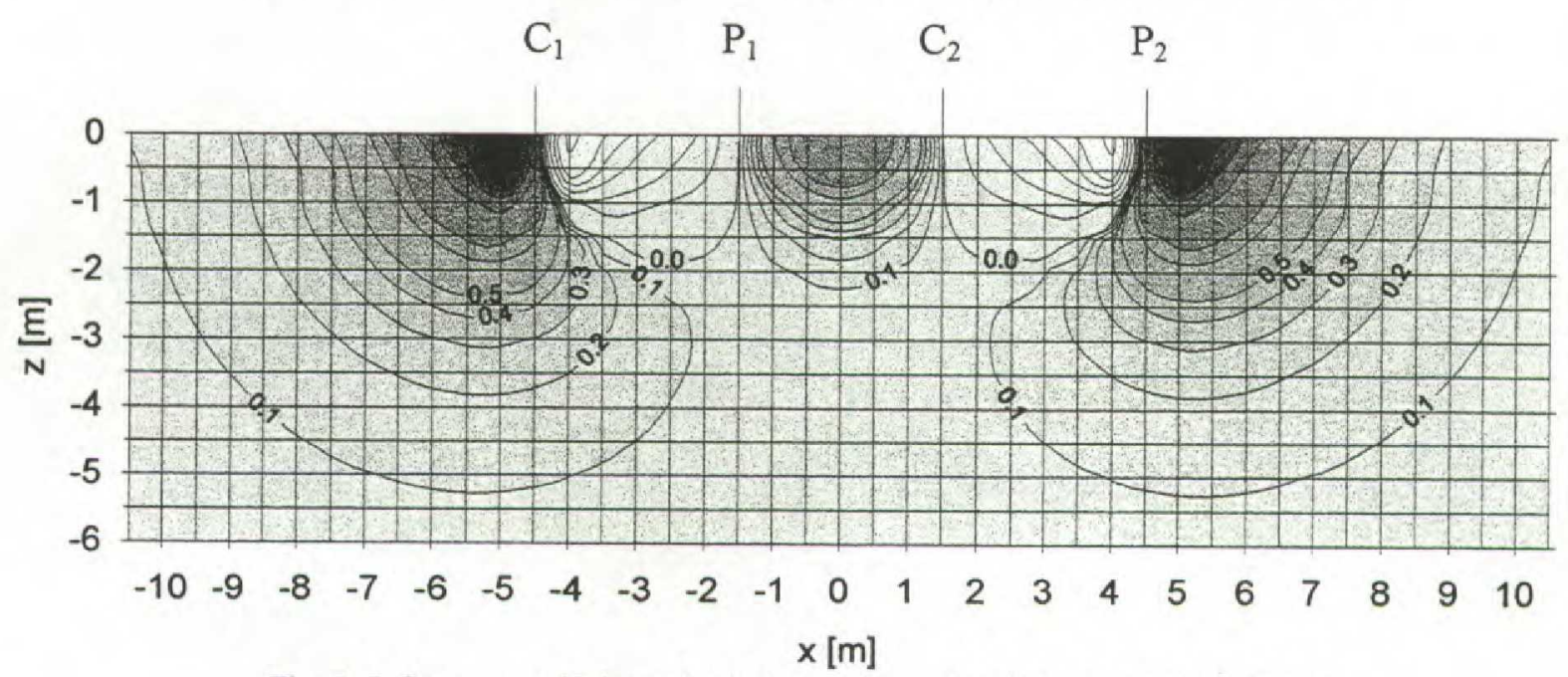

Fig. 3- Influence coefficients for Carpenter array ( $\gamma$ tri-potential) with $P L=6$.

Therefore some tests should be made in order to compute the coefficient to assign the convolution: the assumption of absolute values, as well as the separate computation of positive and negative and subsequent averaging have proved to produce different and useful results (see, for instance, pseudosection of composed resistivity - Cosentino and Luzio, 1994 in fig. 4).

All the tomographic sections have to be subdivided in a number of pixels. It is possible to define a partition level $(P L)$ as the ratio between the smallest distance between two electrodes of the used array (or arrays) and the size of the selected pixel. The choice of $P L$ ratio is important to define both the possible errors due to the partition in a multitude of discrete pixels and the resolution power of the pseudo-section.

The influence coefficient of a pixel generally decreases with its distance from the electrodes: the number of the pixels in which the value of the influence coefficient remains significant enough depends on the $P L$ ratio, being the size of the investigated volume dependent on the size of the electrode array. From a practical point of view the $P L$ ratio should be kept as high as possible to increase the precision and as low as possible to avoid problems of computer memory.

It should be observed that the resolution power depends on various parameters, including the shape, the size the depth and the conductivity contrast of the target to be detected, as well as its direction in the subsoil with respect to the direction of the array. 

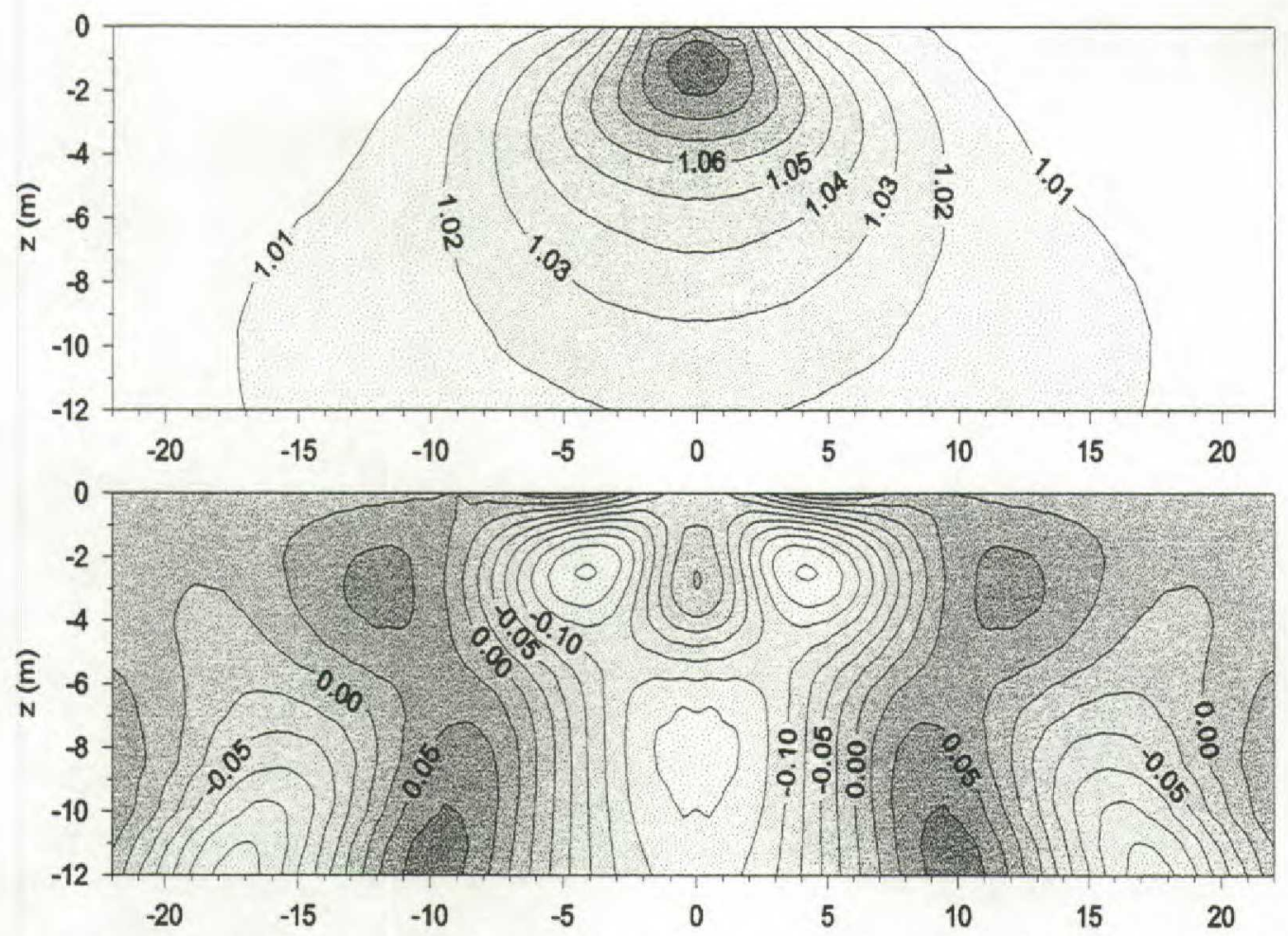

Fig. 4 - Tomographic pseudo-sections of the composed resistivity $\rho_{\mu} / \sqrt{3}=\left(4 \rho_{\alpha}-\rho_{\gamma}\right) / 3$ and $\rho_{\tau}=\sqrt{42}\left(\rho_{\gamma}-\rho_{\alpha}\right)$ for a model of resistive sphere having a radius of $2 \mathrm{~m}$ with the center at a depth of $3 \mathrm{~m}$.

The resolution is however always decreasing with depth and with distance from electrodes, so that the best way to construct a pseudosection should be to use a mesh having the pixels increasing in size with the depth: but this choice is not so immediate also because this behavior depends also on the size of the array as well as the size of the investigated target.

\section{References}

APPARAO, A. (1991): Geoelectric profiling. Geoexploration, 27, 351-389.

APPARAO, A. and V.S. SHARMA (1983): The concept of depth of detection and depth of investigation in resistivity and IP. Geophys. Res. Bull. NGRI (India), 21, 51-61.

COSENTINO, P. and D. LUZIO (1994): Tripotential data processing for H.E.S. interpretation. Annali di Geofisica, 37, suppl. 5, 1295- 1302.

Cosentino, P., D. Luzio, R. Martorana and L. Terranova (1995): Tomographic techniques for pseudo-section representation. Proceedings of the 1st Meeting Environmental and Engineering Geophysics, Torino, 485-488.

EDWARDS, L.S. (1977): A modified pseudosection for resistivity and IP. Geophysics, 42, 1020-1036.

FRASER, D.C. (1981): Contour map presentation of dipole - dipole induced polarization. Geophysical Prospecting, 29, 639-651.

HABBERJAM G.M. (1979): Apparent resistivity observations and the use of square array techniques. Gerbruder Borntraeger, Berlin, 152.

HALlOF, P.G. (1957): On the interpretation of the resistivity and induced polarization measurements. $\mathrm{Ph}$. D. thesis, MIT, Cambridge.

MolleR, I., CHRISTENSEN, N.B., and B.H. JACOBSEN (1995): Fast approximate $2 D$ interpretation of resistivity profile data. Proceedings of the 1st Meeting Environmental and Engineering Geophysics, Torino, 468-471.

ROY, A. and A. APPARAO (1971): Depth of investigation in direct current methods. Geophysics, 36, 943-959. 